\title{
LA CATEGORÍA DE ENERGÍA
}

Las categorías de la ciencia son conceptos en que se expresan, de modo determinado, las formas comunes - y, por ello, más generales- en que se manifiestan las relaciones sociales, las conexiones entre el hombre y la naturaleza y los enlaces internos y externos de los procesos naturales. Las categorías se determinan en el curso de las investigaciones específicas de cada ciencia y se desarrollan y modifican -enriqueciéndose en profundidad y amplitud- con el avance del conocimiento científico. Cuando se investigan dominios de la realidad objetiva que antes no habian sido explorados, la necesidad de modificar las categorías se hace mayor y más urgente, como ocurre en la actualidad en el dominio de los procesos atómicos. En este dominio se han encontrado muchas propiedades que difieren notablemente de las existentes entre los procesos mesocósmicos y los macrocósmicos y, también, se ha descubierto que las formas comunes de existencia de los procesos microcósmicos presentan características bastante extrañas a las expresadas en las categorías de los otros campos de la física. No obstante, son escasas las interpretaciones filosóficas de los problemas planteados por los descubrimientos experimentales de la física atómica $y$, en su mayor parte, se limitan a seguir las orientaciones idealistas contenidas en la "interpretación ortodoxa" de los físicos complementaristas de la Escuela de Copenhague que, como es sabido, no han servido para resolver dichos problemas, ni menos para establecer las nuevas concepciones físicas que son indispensables para explicar, de manera racional y objetiva, el comportamiento de los procesos atómicos. Por esto tiene importancia el hacer un examen crítico de las características que muestra la energía en los distintos niveles de la existencia - $y$, particularmente, en los procesos microcósmicos-, tomando en cuenta los datos aportados por la teoría y la práctica científicas, para poder desentrañar algunos de los elementos necesarios para la integración de esta categoría en el dominio de la física atómica y, en general, de la física entera. $\mathrm{Y}$ esto es justamente lo que nos proponemos realizar aquí.

\section{Canacterización de la energía}

Entre todos los conceptos que la ciencia ha establecido para representar las propiedades de los procesos existentes, la categoría de energía es sin duda el más importante $\mathrm{y}$, a la vez, el más útil. No sólo ha desempeñado, y sigue desempeñando, un papel principal en el desarrollo teórico y experimental de la ciencia, sino que también corresponde a la propiedad física que ha tenido y tiene mayor influencia en el desenvolvimiento de la vida 
humana en todos sus aspectos. En cierto sentido, este desenvolvimiento se podría clasificar históricamente en cuatro etapas - sin que esto signifique el que se excluyan mutuamente-, de acuerdo con las peculiaridades del aprovechamiento humano de la energía: que serían: la de la energía muscular (tanto del hombre como de los animales), la de la energía térmica (obtenida de la leña, del carbón de piedra y del petróleo), la de la energía hidráulica (transformada en varias otras formas) y la de la energía nuclear (en cuyo comienzo nos encontramos ahora). En realidad, el germen de la noción de la energía se pierde en la más remota antigüedad de la historia del hombre. Desde un principio, a la vez que el hombre empezó a desarrollar su propia existencia por medio de su trabajo, aprendió igualmente que la vida humana es imposible sin el trabajo. Más tarde, descubrió el modo de disminuir el esfuerzo que realiza al trabajar -0 , lo que es lo mismo, la manera de aumentar el efecto del esfuerzo realizado- utilizando algunos artefactos mecánicos como la palanca y el plano inclinado. Asimismo, aprendió a acumular su esfuerzo muscular para poder emplear después una fuerza de mayor magnitud en el momento oportuno, con un propósito definido, como ocurre con la invención de la honda y del arco. Luego, logró utilizar también algunas fuerzas de la naturaleza, como el fuego y los animales, para ampliar enormemente los resultados de su esfuerzo en el trabajo. En fin, en el curso de su desenvolvimiento histórico fue encontrando otras fuentes de energía más poderosas y ricas y, a la vez, fue descubriendo modos cada vez más eficaces de aprovecharla. De estas maneras, el hombre comenzb a entrar en posesión consciente de la energía y consiguió imponer su dominio creciente sobre ella.

\section{Determinación científica del movimiento}

Con el surgimiento y la evolución de su actividad científica, el hombre ha podido comprobar que la propiedad más general -a la vez primaria y fundamental - del universo es el movimiento, ya que todos los objetos que lo constituyen, lo mismo que las partes de estos objetos y el conjunto total, se encuentran en movimiento. Esto es, que el universo existe en movimiento o, dicho todavía de otro modo, que la forma primordial de la existencia es e! movimiento. Ahora bien, para representar y estudiar el más simple de los objetos en movimiento, a la vez que los movimientos más sencillos, los físicos formularon el concepto de partícula material. Esta partícula abstracta es considerada como carente de dimensiones, al igual que un punto geométrico, pero ocupa cierta posición en el espacio y puede cambiar de posición con el tiempo. El cambio de posición es un desplazamiento -el más simple de los movimientos-, el ritmo en que cambia de posición en el tiempo es la velocidad $y$, a su vez, la relación en que varía la velocidad con el tiempo es 
la aceleración. Contando con estos elementos conceptuales se pudieron representar también los demás movimientos mecánicos de la partícula material y los movimientos de los otros objetos - considerándolos como sistemas de partículas materiales-, desarrollándose así la cinemática, o sea, la ciencia que estudia abstractamente el movimiento, como el más simple de los cambios espaciotemporales, pero sin atender a las causas que lo provocan y lo modifican; de modo semejante a como la geometría euclidiana se ocupa de estudiar las propiedades espaciales haciendo abstracción del tiempo y, consiguientemente, de las variaciones que produce.

Pero el movimiento es eminentemente dinámico y, por lo tanto, es producido por ciertas causas y, a la vez, es causante de ciertos efectos. Es decir, que el movimiento se produce por la acción de algunas fuerzas y da por resultado cambios en dicha acción. Entonces, la cinemática es ampliada en otra rama de la física, la dinámica, con la cual se consideran las fuerzas que producen el movimiento o que se oponen a éste. Como consecuencia, a la noción de partícula material -siempre manteniendo la abstracción de su carencia de dimensiones- se le agrega la propiedad de tener una masa inercial, que se opone a las fuerzas externas que tratan de impulsarla al movimiento o, mejor dicho, a un cambio en su movimiento. Con estas consideraciones nos encontramos ya en el dominio de la mecánica propiamente dicha. Ahora bien, en todo movimiento se muestran dos aspectos opuestos: la acción o impulso, y la reacción o resistencia. En rigor, el movimiento se manifiesta como resultante del conflicto entre la acción y la reacción; y, así, hasta el reposo de un objeto viene a ser sencillamente un caso particular del movimiento, que se produce como resultado del equilibrio entre la acción y la reaccion. La resistencia o reacción es lo que se llama masa, o aspecto positivo; mientras que el impulso o acción es denominado fuerza - que viene a ser la manifestación elemental de la energía. Pero ambos aspectos son inseparables y, además, se intercambian entre sí y se transforman recíprocamente uno en otro. La masa ejerce presión $y$, por consiguiente, se muestra como fuerza. Igualmente, la fuerza llega a colocarse en condiciones de oponerse al movimiento y, en este sentido, se manifiesta como masa resistente. Además, como ex sabldo, Einstein descubrib la proporción en que masa y energía se conviertea mutuamente, una en otra y viceversa, expresándola en su famosa acuación: $E=m c^{2}$ (en donde, $E$ es la energía, $m$ la masa y $c$ la velocidad de la haz). Como la velocidad de la luz es constante -ya que es el límite máximo de la velocidad a que se transmite la energía- y su valor es de $300000 \mathrm{~km} / \mathrm{seg}$, entonces, entre la masa y la energía de un objeto hay una relación tal que, un aumento en la energía se obtiene a costa de la transformación de una parte de la masa y, recíprocamente, la masa crece por la conversión de la energía. Sólo que, mientras la energía crece de esta manera 
en proporción de $9 \times 10^{20}$ veces la masa, ${ }^{1}$ en cambio, la masa aumenta en ia relación de $11 \times 10^{-22}$ veces la energía. ${ }^{2}$

\section{La energía mecánica}

En la física, la masa de los objetos se define estableciendo que la relación entre las masas de dos cuerpos que se encuentran en interacción es igual a la razón inversa de las aceleraciones resultantes en ellos por dicha interacción. Tomando como unidad la masa de un cierto objeto, es posible determinar - al menos, en principio- la masa de cualquier otro objeto del universo. Con esta manera de determinar cuantitativamente la masa, y teniendo en cuenta el hecho de que toda acción produce una reacción igual y contraria, se establece la definición cuantitativa de la fuerza, como el producto de la masa por la aceleración. Pues bien, como ya lo dijimos antes, la concepción de la energía se formó históricamente a partir del trabajo, ya que, trasladando su noción a la física y considerándolo cuantitativamente, vieno a ser simplemente la medida de lo que puede llamarse el efecto espacial de una fuerza sobre un objeto. Así, por ejemplo, el trabajo ejecutado por una fuerza al actuar sobre un objeto y hacerlo que se desplace, se define como el producto de la fuerza por la longitud del desplazamiento. Si consideramos dos partículas $A$ y $B$, entre las cuales se ejerce mutuamente una fuerza, tenemos que $A$ actúa sobre $B$ con una fuerza que es igual y opuesta a la que $B$ ejerce sobre $A$. Si se aplica la ley newtoniana del movimiento a cada una de estas partículas, según la cual la fuerza es igual al producto de la masa por la aceleración, se puede calcular fácilmente el trabajo ejecutado por la fuerza mutua, cuando las partículas se mueven relativamente entre sí. En general, para un cierto intervalo de tiempo, este trabajo no es el mismo para las dos partículas, ya que su desplazamiento dependerá de la magnitud de su masa. Y como lo más común será que las partículas tengan masas diferentes, entonces se comportarán de distinta manera, ya que tendrán aceleraciones y velocidades diferentes. Sin embargo, si se suman las respectivas expresiones del trabajo de cada partícula, haciendo una reordenación algebraica sencilla, se obtiene un resultado sorprendente, ya que, a pesar de la complejidad que puedan tener los movimientos de las partículas, existe una expresión muy bien definida que permanece constante durante dichos movimientos y que incluye la posición espacial, la velocidad, la masa y la fuerza de ambas partículas. Y lo mismo ocurre para un número cualquiera de partículas, por grande que sea este número siempre permanecerá constante tal expresión. Pues bien, esta invariante del movimiento es lo que se denomina, en la física,

1 Esto es, en la proporción de 900000000000000000000 veces la masa.

2 O sea, que una unidad de masa se convierte en 0.0000000000000000000011 unidades de energía. 
energía mecánica. En rigor, esta energía consta de dos partes: la energía cinética, que depende de la masa y de la velocidad de cada partícula; y la energía potencial, que depende de las posiciones relativas en que se encuentran las partículas.

Adoptando otro enfoque importante, podemos advertir que el movimiento muestra también dos aspectos opuestos: la atracción y la repulsión. En sentido estricto, el movimiento es el resultado de la oposición entre la atracción y la repulsión. Así, por ejemplo, el movimiento de los planetas en el sistema solar es sencillamente el resultado del equilibrio dinámico establecido entre la atracción centrípeta que el sol ejerce sobre los planetas -debida a la gravitación universal- y la repulsión centrífuga que los planetas ejercen por su parte -que se debe al movimiento de su masa. En este caso de oposición entre aspectos del movimiento, la atracción es llamada fuerza porque representa el aspecto pasivo - sobre todo en las condiciones en que vive el hombre en la Tierra, en donde la atracción gravitatoria del Sol predomina hasta el punto de que únicamente se percibe cuando se manifiesta una repulsión particular y transitoria-, en tanto que la repulsión es denominada energía, porque es el aspecto que más se hace notar. Cuando un movimiento cualquiera se transfiere de un cuerpo a otro, se le considera como activo, en tanto que se transmite $y$ es causa del otro movimiento; $o$, dicho de otro modo, en tanto que produce movimiento en el otro cuerpo. Pero, simultáneamente, en tanto que es transmitido, el mismo movimiento es pasivo y su causa - que es el movimiento activo- se muestra como energía, de la cual el movimiento pasivo es considerado como una manifestación. No obstante, la energía es exactamente de la misma magnitud que su manifestación, ya que se trata simplemente de dos aspectos de uno y el mismo movimiento.

\section{La medida del movimiento}

En el siglo xur se inició una famosa controversia acerca de cuál era la relación entre magnitudes físicas que debería ser considerada como medida del movimiento. Descartes sostuvo que la cantidad de movimiento total que existe en el universo es constante - como se sabe, la cantidad de movimiento define cuantitativamente como el producto de la masa de un cuerpo por su velocidad, o sea, mv- y, más aún, que todos los cambios que ocurren en los procesos son resultado de las transferencias de cantidad de movimiento de tmas partículas a otras, cuando existe interacción entre ellas. Por ende, para Descartes, la verdadera medida de la fuerza se encuentra en el cambio de la tuntidad de movimiento producido en un tiempo dado. De este modo, Descartes formulo por primera vez el principio de la conservación de la cantidad de movimiento. Sin embargo, esta formulación era imprecisa, porque Descartes consideraba a la velocidad como un simple escalar -esto es, como un 
simple número con magnitud- sin tomar en cuenta que también tiene dirección y sentido y que, por lo tanto, es un vector.

En 1668, la Royal Society de Londres organizó una encuesta sobre este problema de la medida del movimiento. John Wallis aportó la justa observación de que era necesario considerar con signos contrarios a las velocidades opuestas, con lo cual introdujo la consideración de los dos sentidos que puede tener una velocidad en una misma dirección. Christiaan Huyghens, por su parte, demostró correctamente que no sólo la suma de las cantidades de movimiento - considerando ya en el producto $m v$ los signos + y - de la velocidad- tiene el mismo valor antes y después de un choque perfectamente elástico entre dos o más partículas, sino que también es constante la suma de los productos de las masas respectivas por los cuadrados de las velocidades asociadas a ellas, esto es, la suma de los productos $m v^{2}$. Además, Huyghens utilizó el principio galileano de relatividad, para la transformación de un sistema de referencia a otro que se mueve con velocidad uniforme respecto al primero. Con la introducción de estas acertadas consideraciones de Huyghens se tuvieron realmente los elementos suficientes para poder resolver el problema de la medida del movimiento.

Newton encontró que el ritmo de cambio de la cantidad de movimiento lineal de una partícula material, por unidad de tiempo, es igual a la fuerza que actúa sobre dicha partícula. Y de aquí, considerando también una de las leyes del movimiento formuladas por el propio Newton -la de que las fuerzas que se ejercen entre dos partículas materiales son iguales y opuestas- llegó a establecer que las interacciones ejercidas entre un número cualquiera de partículas materiales no cambia nunca la cantidad de movimiento total de un sistema, con tal que éste no se encuentre sometido a fuerzas extrañas al propio sistema. Para calcular la cantidad de movimiento total, Newton tomb́ justamente esta magnitud física como un vector $y$, en consecuencia, sumb́ vectorialmente las cantidades de movimiento individuales. En este caso, en vez de considerar a la fuerza como el producto de la masa por la aceleración, la igualó con el ritmo de cambio de la cantidad de movimiento; lo cual viene a ser equivalente si la cantidad de movimiento es considerada como el producto de la masa por la velocidad. Esta formulación newtoniana de la ley de conservación de la cantidad de movimiento constituye una expresión tan profunda y objetiva, que ha resultado consecuente con la física relativista y con la mecánica cuántica; mientras que la otra formulación solamente mantiene su validez dentro del dominio de la física clásica. ${ }^{3}$

Leibniz fue el primero en llamar la atención acerca del producto de la masa por el cuadrado de la velocidad, $m v^{2}$, al que le dio el nombre de vis viva -o sea, fuerza viva-y que viene a ser el doble de lo que después se

3 Esta limitación quedó demostrada experimentalmente por la deflexión que sufren los electrones que se mueven a velocidades comparables a la de la luz. 
llamó energía cinética. Según Leibniz, la genuina medida de la eficacia de una fuerza es precisamente dicha vis viva. Esta divergencia con Descartes dio lugar a la larga y apasionada polémica a que antes nos referíamos, entre los leibnizianos y los cartesianos, en torno al problema de saber cuál era la medida correcta de la fuerza ejercida por el movimiento. En realidad, la disputa entrañaba una confusión terminológica, provocada por la ambigua significación que se daba entonces al concepto de fuerza. D'Alembert pudo resolver la controversia, demostrando que el efecto acumulativo de una fuerza se puede medir por su efecto integrado sobre el tiempo -esto es, por el cambio que produce en la cantidad de movimiento ${ }^{5}$, como por su efecto integrado sobre el espacio -o sea, el cambio que produce en la energía cinética ${ }^{6}$ Ambas medidas del movimiento son entonces igualmente válidas, pudiéndose escoger libremente una u otra, de acuerdo con la conveniencia del problema concreto que se trate de resolver.

Desde un punto de vista moderno, el problema crítico que surgió en la controversia acerca de la medida del movimiento, fue el de saber cuál era el principio fundamental para explicar todos los procesos físicos: el principio de la conservación de la cantidad de movimiento, o el principio de la conservación de la energía. En cierto modo, dentro del dominio de la mecánica, el principio que acabó por triunfar fue el de la conservación de la cantidad de movimiento, puesto que se cumple estrictamente en todas las interacciones de los cuerpos, ya se trate de las dimensiones astronómicas, de las comparables al hombre o de las ultramicroscópicas. Pero, al mismo tiempo, el principio de la conservación de la energía es el que ha permitido explicar justamente la naturaleza de las fuerzas que intervienen en dichas interacciones $y$, lo que es más, este principio ha superado los límites de la consideración mecanicista y ha mostrado su vigencia en todos los campos de la física, incluyendo tanto el mesocosmos como el macrocosmos y el microcosmos. A la vez, al generalizarse la concepción de la energía a la física entera - por imposición de la realidad objetiva- se descubrieron otras formas de energía - térmica, eléctrica, magnética, luminosa, radiante en general, nuclear, etc.- que son diferentes a la energía cinética y a la energía potencial de la mecánica. Por otra parte, también se ha descubierto que todas las distintas formas de energía se transforman mutuamente unas en otras, en relaciones equivalentes que han

4 G. G. Coriolis fue quien aplicó por primera vez el valor $\frac{1}{2} \mathrm{mv}^{2}$; y Johannes Bernoulli fue el primero en utilizar la palabra energía, aunque su empleo se vino a generalizar hasta más tarde.

5 Dimensionalmente, la cantidad de movimiento (en unidades cgs: centímetro por gramo entre segundo, $\mathrm{cgs}^{-1}$ ) es igual a la fuerza (centímetro por gramo entre segundo al cuadrado, cgs-2) multiplicada por el tiempo (segundo, s).

- También desde el punto de vista dimensional, la energía (centímetro al cuadrado por gramo entre segundo al cuadrado, $c^{2} g_{s}-2$ ) es igual a la fuerza (centímetro por gramo entre segundo al cuadrado, $\mathrm{cgs}^{-2}$ ) multiplicada por la longitud (centímetro, $c$ ). 
sido determinadas con exactitud. De este modo, no solamente se conserva objetivamente la energía, sino que las diversas formas de su existencia se convierten recíprocamente unas en otras.

\section{Conservación y transformación de la energía}

De acuerdo con la física contemporánea, podemos decir que en todos los objetos de la naturaleza existe una propiedad a la que denominamos energía, que puede ser considerada como la capacidad para producir efectos y que tiene la peculiaridad de que, aunque se presenta en varias formas y es susceptible de distintas transformaciones, sin embargo, su cantidad total no cambia y, por lo tanto, siempre se conserva constante. En el caso de la energía mecánica, su efecto es la ejecución de un trabajo; en el caso de la energía electromagnética, su efecto es un flujo de energía electromagnética; en el caso de la energía térmica, su efecto es la transmisión de calor; y así sucesivamente. Por lo demás, como cada forma de energía es susceptible de convertirse en cualquier otra de sus formas, resulta que, de un modo directo o indirecto, cada una de las formas de energía es capaz de producir todos esos efectos de manera equivalente. En fin, como la masa y la energía se transforman también mutuamente, en la proporción expresada por la ecuación de Einstein ( $E=m c^{2}$ ), tenemos que la energía produce igualmente los efectos de la masa $y$, viceversa, que la masa es capaz de transferirse y manifestarse en alguna de las formas de la energía.

Aquí cabe hacer una advertencia sumamente importante, para evitar dos errores que se cometen con frecuencia. En primer lugar, no se debe confundir la masa con la materia - ya que, en todo caso, la masa es solamente una de las muchas propiedades de la materia, esto es, de la existencia objetiva$y$, por consiguiente, es una interpretación insostenible la que se hace al hablar de que "la materia desaparece" cuando ocurre una conversión de masa en energía. En realidad, la energía es tań material como la masa, ya que es otra de las propiedades de la materia y, por lo tanto, todas sus manifestaciones son tan objetivas y susceptibles de experimentación como lo son las manifestaciones de la masa. Además, nunca se debe olvidar que la conversión descubierta por Einstein es recíproca y, en consecuencia, que la energía también se transforma en masa, sin desaparecer tampoco por esto. Por otro lado, aun cuando existen diversas formas de energía, no por ello hay que atribuir a cada proceso existente una forma particular de energía para poder explicarlo, ni menos hay que considerar en caso alguno una especie de energía tal que se supusiera que tuviese existencia aparte de la materia. Si se multiplica innecesariamente el número de formas de energía, no sólo se procede con plena arbitrariedad subjetiva, sino que se acaba por abandonar definitivamente la explicación científica. Por todo esto, lo que realmente preocupa al cien- 
tífico es investigar con el mayor rigor - tanto teórica como experimentalmepte- todos y cada uno de los procesos existentes, hasta descubrir objetivamente la forma de energía, o la conjugación de varias formas de energía, que produce efectivamente los movimientos y los cambios, por complejos que éstos sean. De otra manera, cuando se inventan formas de energía inexistentes o supuestamente inmateriales, lo que se consigue es abandonar en definitiva el terreno de la ciencia, para caer de lleno en la especulación sin base.

\section{Principios de conservación}

La tarea primordial de la física consiste en descubrir las leyes generales de la naturaleza y en expresarlas de manera racional y objetiva, para poder fundar sobre ellas la explicación de los procesos físicos conocidos experimentalmente y anticipar hipotéticamente la existencia de otros procesos y su comportamiento. Ahora bien, entre las leyes generales más profundas y de más simple expresión, tenemos a los principios que expresan la conservación de alguna de las propiedades universales de los procesos existentes. Por ello, la búsqueda de la constancia en la magnitud total de ciertas propiedades físicas es una preocupación legítima que tiene gran importancia para los investigadores científicos $y$, algunas veces, a ella se ha debido el que se adquiera tempranamente la convicción de que la cantidad total de cierta propiedad física se mantiene constante, como ocurrió, por ejemplo, en el caso de la carga eléctrica. Sin embargo, para que un principio de conservación se convierta efectivamente en una ley de la física, es enteramente indispensable que su postulación sea verificada de un modo incontrovertible a través de la experimentación, que fue lo que finalmente se logró respecto a la conservación de la eléctricidad. De manera correspondiente, también la refutación experimental es lo único que puede llevar a demostrar el incumplimiento o la limitación de un principio de conservación, como ha sucedido recientemente con la falla de la conservación de la paridad y la violación de la invariancia de la conjugación de la carga en las interacciones débiles de las partículas elementales, puestas al descubierto por los trabajos de Yang Chen-ning y Lee Tsung-dao. ${ }^{7}$

En la física contemporánea, las leyes de conservación han adquirido una importancia todavía mayor de la que tenían en la física clásica, debido principalmente al hecho fundamental de que se ha probado que su validez se mantiene inalterablemente en los dominios de la física relativista y de la física coàntica, a diferencia de otras muchas leyes de la física clásica que han tenido que sufrir modificaciones radicales para poder ser aplicables a es-

7 Venos as discursos de recepción del Premio Nobel de Física, en Simetría y pa6ut 801. Entroudad Nacional de México, 1958. 
tos nuevos campos del conocimiento o que, sencillamente, carecen de validez en estos niveles de la existencia objetiva. Por otra parte, cada ley de conservación se encuentra conectada en forma indisoluble y recíproca con una ley de simetría o, lo que es equivalente, con un principio de invariancia. Así, por ejemplo, existe una correspondencia objetiva y biunívoca entre la conservación de la cantidad de movimiento lineal y la invariancia de las leyes físicas ante una translación espacial. Además, esta conexión entre la simetría y la conservación se ha ampliado notablemente por el hecho de que, mientras en la mecánica clásica únicamente se cumple para las simetrías continuas -como son la translación, la rotación y las transformaciones de Galileo y de Lorentz, por ejemplo-, en cambio, en la mecánica cuántica también tiene cumplimiento para las simetrías discretas - como son, por ejemplo, la reflexión es. pacial y la inversión temporal.

Considerando el dominio entero de la física —o sea, el dominio de la física relativista, el de la mecánica cuántica $y$, por consiguiente, también el de la física clásica en que coinciden la cuántica y la relativista- tenemos que, fundamentalmente, existen tres leyes de conservación: el principio de inercia, que implica la conservación de la cantidad de movimiento lineal; el principio de conservación de la energía, a través de la transformación en todas sus formas de existencia; y el principio de conservación de la cantidad de electricidad. También existen otras dos leyes de conservación, que son: el principio de conservación de la cantidad de movimiento angular; y el principio de conservación de la masa inerte. Pero la primera de estas dos últimas leyes resulta ser, en cierto modo, una consecuencia directa del principio de conservación de la cantidad de movimiento lineal; $y$, lo que es más, si consideramos conjuntamente la translación y la rotación - que son los movimientos elementales, desde el punto de vista espacial-, entonces ambas leyes quedan conjugadas en el principio de conservación de la cantidad de movimiento (tanto angular como lineal). Por su parte, con el descubrimiento de la conversión mutua entre la masa y la energía, la ley de conservación de la masa inerte ha quedado incluida dentro del principio más general de la conservación y la transformación de la energía y la masa. Por otro lado, en la física relativista, se han fundido en una sola ley los principios de la conservación de la energía y de la conservación de la cantidad de movimiento. En fin, en el dominio de la física cuántica tenemos otros principios de conservación -como son los que establecen la conservación de la paridad, de las partículas pesadas o bariones, del spin isotópico, y de las partículas livianas o leptones- cuyo verdadero carácter y alcance son estudiados intensamente en la actualidad, tanto en forma teórica como experimental.

En el caso de los procesos atómicos, son bien conocidas las graves dificultades que surgen cuando se trata de prever con exactitud el comportamiento individual de las partículas elementales. En general, la teoría cuántica "or- 
todoxa" sólo permite determinar con cierto rigor $-\mathrm{y}$ en esto radica una de sus mayores insuficiencias, aunque no es la única- el comportamiento de grandes conjuntos de partículas, estableciendo las probabilidades correspondientes a los diversos resultados que son posibles. Sin embargo, hay algunas leyes físicas que sí permiten establecer con entera precisión el comportamiento de las partículas individuales. Y entre estas leyes se encuentran justamente los principios de conservación. Debido a este cumplimiento universal que tienen las leyes de conservación en todos los niveles de la existencia, es por lo que uno de los criterios para juzgar acerca de la estructura lógica de cualquier tearia física nueva consiste en indagar si es consecuente con los principios de conservación y, especialmente, con la ley de la conservación y la transformación de la energía y la masa. No obstante, es necesario aclarar que la aplicación de este criterio científico es algo completamente diferente a lo que significa la adopción de la infundada "concepción energética" de Ostwald. En realidad, los principios de conservación tienen -como todas las leyes cientificas- el carácter de teorías generales comprobadas $y$, por consiguiente, son los fundamentos en que se apoya toda la estructura de los conocimientos adquiridos. Pero en todo caso, respecto a las nuevas investigaciones, las leyes científicas mantienen su carácter de postulados elementales por verificar, aunque estén apoyadas en su comprobación reiterada e inexcepcional en todos los conocimientos anteriores. Y solamente se convierten también en fundamentos de los nuevos conocimientos, cuando se consigue comprobar experimentalmente que dichas leyes también se cumplen en los conocimientos nuevos. Unicamente con estas exigencias se asegura la objetividad de los resultados logrados en la experimentación y en el desarrollo teórico de la ciencia.

\section{La inercia de la energía}

Cuando una carga eléctrica se desplaza, el movimiento del campo eléctrico correspondiente produce el surgimiento de un campo magnético $\mathrm{y}$, entonces, su coexistencia trae aparejados un flujo de energía y una cantidad de movimiento adicional. Si el sistema satisface ciertas condiciones de simetría -por ejemplo, si se trata de una esfera-, la energía y la cantidad de movimiento quedan representados por dos vectores que son paralelos al vector que representa la velocidad. De este modo, nos encontramos con la existencia de la inencia de la energía electromagnética, ya que su efecto es enteramente semejante al producido por la inercia de la masa; o sea, que la cantidad de movimiento adicional implica ineludiblemente una masa inercial adicional. Para un cuerpo macroscópico, esta masa adicional es despreciable en comparación con su masa en reposo, en el caso de todas las cargas que son manejables experimentalmente. Pero cuando se trata de una partícula elemental como, por ejemplo, un electrón -que puede ser considerado, en muchos respectos, 
como una esfera rígida-, esta masa adicional resulta ser del mismo orden de magnitud que su masa en reposo. En 1903, Max Abraham encontró que la cantidad de movimiento debida a la energfa electromagnética es proporcional a la velocidad, pero solamente en el caso de velocidades relativamente muy pequeñas; en cambio, dicha cantidad de movimiento aumenta mucho más rápidamente -de hecho, sin límite alguno- a medida que la velocidad del electrón se aproxima a $c$, la velocidad de la luz. Este resultado puso en crisis la estructura básica de la mecánica clásica, planteando la necesidad de establecer una nueva teoría, que fue justamente la teoría de la relatividad. Einstein, en 1905, puso al descubierto la fundamental relación existente entre la masa $m$ y la energía $E$, conduciendo así al reconocimiento de que todas las formas de energía poseen inercia. Al determinar la inercia de la energía, la teoría de la relatividad estableció la conjugación entre la densidad de la energía, el flujo de energía y la densidad de la cantidad de movimiento, en una sola magnitud física que se expresa matemáticamente por medio del tensor energía-cantidad de movimiento. De esta manera fue como quedaron fundidos en una sola ley los principios de la conservación de la cantidad de movimiento y de la conservación de la energía. Y la validez de esta fusión, conseguida primero teóricamente recurriendo al principio de relatividad, ha sido verificada después a través de la experimentación.

La ley generalizada de la inercia de la energía implica necesariamente el tener que reconocer, por un lado, que la masa y la energía son dos formas diferentes e inseparables, en las cuales se manifiesta la existencia de los procesos objetivos; $y$, por otra parte, que la cantidad de movimiento y el flujo de energía son igualmente dos aspectos distintos e inseparables de un mismo modo de existencia de dichos procesos. Así, tanto la masa como la energía han dejado de ser categorías independientes de la física, para quedar sintetizadas en la nueva categoría de energía-masa, que es mucho más rica en contenido que la suma de todas las propiedades de la masa y de todas las características de la energía. En particular, la concepción de la energía se ha ampliado enormemente como resultado de su conjugación dialéctica con la masa. Desde luego, no obstante que hay algunas buenas razones para considerar que tal vez no conocemos todavía todas las formas en que existe la energía, el hecho es que, independientemente de esto, en la actualidad podemos determinar la cantidad total de energía que posee un objeto físico, partiendo de la magnitud de su masa. Por lo tanto, ha desaparecido la exigencia de tener que fijar arbitrariamente un punto cero para la energía, cosa que la concepción anterior obligaba a introducir; y, entonces, resulta que ya no se consideran meramente diferencias de energía, como antes ocurría, sino que la energía representa una magnitud física bien determinada. Ahora bien, teniendo en cuenta las conjugaciones que hemos mencionado, la teoría de la relatividad puede ser considerada como la teoría del espacio y del tiempo 
que refleja la existencia de la conexión mutua universal entre la masa y la energía. Y, por consiguiente, del mismo modo que todo cuerpo posee extensión espacial y todo proceso tiene duración temporal, así también todo cuerpo tiene una masa en reposo, $m_{0}$, y una energía propia no relativa, $m_{0} c^{2}$, o sea, más precisamente, una magnitud compleja a la que denominamos energíacantidad de movimiento.

\section{Temporalidad de la energía}

El examen riguroso de las relaciones de incertidumbre de Heisenberg ${ }^{8}$ nos ha permitido establecer que la consideración simultánea de las magnitudes representadas por los conceptos de "energía precisamente determinada" y de "instante precisamente determinado", respecto a una partícula elemental, conduce a la conexión lógica que se expresa por medio de un juicio de incompatibilidad. Por lo tanto, en tal caso existen tres posibilidades: a) la determinación precisa de la energía, a costa de la determinación precisa del tiempo; b) la determinación precisa del tiempo, en detrimento de la determinación precisa de la energía; y c) la determinación simultánea de la energía y el tiempo con la precisión permitida por el límite expresado justamente por la relación de incertidumbre. De otra parte, también tenemos que en una de las ecuaciones primordiales de la mecánica cuántica, la energía $E$ de una partícula elemental queda determinada exactamente por la constante universal de Planck, $h$, multiplicada por la frecuencia $v$ de su movimiento ondulatorio -o sea, el número de crestas de onda que pasan por un punto en la unidad de tiempo- de acuerdo con la relación: $E=h v$. Esta ecuación impone la necesidad de operar simultáneamente con la energía y con una propiedad temporal - como lo es la frecuencia, que viene a ser dimensionalmente el inverso del tiempo- de una y la misma partícula elemental. Y esta operacion requiere ineludiblemente el tomar valores precisos de ambas magnitudes. Sólo que, conforme a la relación de incertidumbre correspondiente, esto es justamente imposible hacerlo con precisión respecto a la energía y el tiempo. De esta manera, se plantea una paradoja insostenible: de un lado, la imposibilidad de hacer una determinación precisa y simultánea de la energía y el tiempo; de otra parte, la imposibilidad de eludir la necesaria determinzción simultánea y precisa de la energía y la frecuencia, que también es una propiedad temporal.9

Ahora bien, el hecho mismo de que la oposición antes señalada se muestre como irreductible, plantea la necesidad de tratar de superar dialécticamente

8 Véase nuestro ensayo "El tiempo en la física atómica", Dínoia, IV, 1958; sec. 1, págs. 64-66.

- Esto mismo sucede para las otras parejas de magnitudes afectadas por las relaciones de incertidumbre. Véase nuestro ensayo "La categoría de espacio en la física atómica", Dínoia, III, 1957; secs. 5 y 7, págs. 105-111 y 113-115. 
esta paradoja, con el fin de resolverla. Para esto, tenemos que considerar la energía de una partícula elemental como otra forma en la que se manifiesta su oscilación ondulatoria o frecuencia; y, a la vez, tenemos que reconocer en la oscilación ondulatoria de su movimiento otra manera en la cual se muestra su energía. Entonces, el tiempo tiene que ser considerado conjuntamente como energía y como oscilación; o bien, dicho de otra manera, se tiene que reconocer a la energía y a la frecuencia como dos formas, distintas pero inseparables, a través de las cuales se manifiesta un modo de existencia de las partículas atómicas, al que denominamos genéricamente como sus propiedades temporales. Por lo demás, la objetividad de esta conjugación entre el tiempo y la energía ya ha sido demostrada antes en el dominio de la física relativista. Como consecuencia, viene a resultar conceptualmente posible y experimentalmente necesario el determinar a la vez con precisión, para una y la misma partícula, la magnitud de su energía y de una de sus propiedades temporales. Más aún, la relación lógica entre estas magnitudes la podemos expresar por medio de un juicio de inclusión. Por lo tanto, tendría este enunciado: Respecto a las partículas elementales, el tiempo se puede manifestar como oscilación, o como energía, o bien, como oscilación y energía a la vez. De esta manera quedan satisfechas, rigurosamente, tanto la relación de incertidumbre para la pareja energía-tiempo, como la ecuación que conjuga la frecuencia del movimiento ondulatorio con la energía; lo cual es posible, objetivamente, por la coincidencia de la constante cuántica $h$ en ambas expresiones.

\section{Energía interna de los atomos}

Como es sabido, cada átomo constituye un sistema dinámico formado por un núcleo - que es simple únicamente en el caso del hidrógeno ordinario y compuesto en todos los demás, desde el deuterio hasta el nobelio, pero cuyo comportamiento se puede considerar, para muchos efectos, como el de una partícula unitaria con carga positiva - y por un cierto número de electrones negativos, que pueden ser desde 1 hasta 102, coincidiendo justamente con el número atómico del elemento químico al cual corresponde. Dentro de este sistema atómico, el núcleo permanece casi fijo y los electrones se mueven alrededor suyo, describiendo trayectorias bastante complejas. Como ya lo hemos dicho, dentro de este sistema se mantienen los principios de conservación de la energía, de conservación de la carga eléctrica y de conservación de la cantidad de movimiento; $y$, por consiguiente, su energía total permanece constante. Desde el punto de vista mecánico, dicha energía total está formada por la suma de la energía cinética del núcleo, de la energía cinética de los electrones y de la energía potencial mutua. Pero, debido a que la masa del núcleo es enormemente mayor que la del electrón, el centro del núcleo coinc: 
de aproximadamente en todos los instantes con el centro de gravedad del átomo y como, por otra parte, el núcleo casi no se mueve, resulta posible el despreciar prácticamente la contribución del núcleo a la energía cinética del sistema. De aquí que podamos considerar, con una gran aproximación, que la energía total del sistema formado por un átomo sea igual a la suma de las energías cinéticas de sus electrones planetarios y de sus respectivas energías potenciales.

Ahora bien, para poder explicar satisfactoriamente los procesos de absorción y de emisión de energía radiante que se producen en el átomo, fue necesario reconocer la existencia de limitaciones en el movimiento de los electrones alrededor del núcleo, de tal modo que únicamente son posibles ciertas órbitas privilegiadas, denominadas órbitas estables o cuánticas, cuyos radios forman una serie infinita pero discreta de valores. Esta condición -propuesta originalmente por Bohr, en 1913, y verificada después experimentalmente- consiste en limitar los radios posibles a aquellos en los cuales la cantidad de movimiento angular del electrón resulta ser un múltiplo entero de la cantidad cuántica $\frac{h}{4 \pi}$, en donde $h$ es la constante de Planck y $\pi$ es la conocida relación entre el diámetro y la circunferencia. ${ }^{10}$ En el caso del átomo de hidrógeno, la órbita más cercana al núcleo tiene un radio de $0.527 \times 10^{-8} \mathrm{~cm}$.; $y$, tomando este valor como unidad, la serie de los radios posibles para las órbitas es la de los cuadrados de los números naturales, o sea, 1, 4, 9, 16, $25, \ldots$ A cada una de estas órbitas le corresponde una cantidad definida de energía $y$, por lo tanto, esta energía de los electrones sólo puede asumir valores discretos, llamados niveles de energía. A su vez, cada uno de estos niveles de energía corresponde a un estado cuántico diferente para el electrón y, como vamos a ver luego, también para el átomo. Por otra parte, la consideración ondulatoria del movimiento de los electrones conduce a poner al descubierto que sus trayectorias dentro del átomo tienen necesariamente longitudes tales que son múltiplos enteros de la longitud de onda de los propios electrones y que, además, coinciden precisamente con las órbitas correspondientes a los niveles de energía que resultan posibles cuánticamente. En consecuencia, la localización espacial y temporal de las órbitas electrónicas se encuentra especificada por la cuantización de la cantidad de movimiento angular, por la cuantización de la energía y por la dimensión finita de la longitud de onda de los propios electrones.

El paso de un electrón de una órbita cuántica a otra se produce siempre de manera brusca, en la forma de un salto cuántico. Y, como se cumplen los principios de conservación de la cantidad de movimiento y de la energía, para que se produzca uno de estos saltos es indispensable que el átomo

10 Como es fácil advertir, se trata de la misma magnitud constante que expresa el limite de la precisión que es posible, de acuerdo con las relaciones de incertidumbre. 
absorba o emita la cantidad de energía correspondiente a la diferencia entre. los dos niveles en cuestión. Esta energía es absorbida o emitida en la forma de radiación; habiendo absorción de energía cuando la transición del electrón es de una órbita interna a un nivel externo de mayor energía, y emisión en el caso contrario. Debido a que la masa total del átomo es enorme, comparada con la del electrón, la energía cinética que un átomo puede recibir cuando choca con un electrón libre es enteramente despreciable respecto al átomo considerado en su conjunto. Por ende, toda la energía transferida al átomo por el choque viene a incrementar su energía interna. Cuando la energía del electrón libre que choca es menor que la requerida para provocar el paso de un electrón atómico a una órbita exterior -esto es, cuando es menor que la energía de resonancia-, entonces el átomo no sufre alteración interna alguna y el electrón libre sale del choque como un cuerpo perfectamente elástico. ${ }^{11}$ En cambio, cuando la energía del electrón libre que choca es un poco mayor que la energía de resonancia, su absorción puede provocar el salto de un electrón atómico a una órbita exterior a su trayectoria básica, haciendo que el átomo deje de encontrarse en su estado estable y pase a un estado de excitación. Este estado de excitación tiene una duración sumamente breve y termina cuando el electrón excitado vuelve a su órbita estable y el átomo emite la energía correspondiente en forma de radiación. Por su parte, el electrón libre sale despedido del choque con una energía cinética que es menor de la que traía, exactamente en la diferencia entre su energía inicial y la energía de resonancia absorbida por el átomo; de este modo, ocurre como si el electrón hubiera chocado con un cuerpo blando y, por ello, la colisión se llama entonces inelástica.

También puede suceder que la energía cinética del electrón libre que choca con un átomo sea mayor todavía a la antes indicada $y$, en ese caso, se producen choques inelásticos que hacen que el átomo pase sucesivamente a las siguientes energías de excitación. En tales casos, el electrón libre sale despedido con una energía cinética disminuida exactamente en la cantidad transferida al átomo. Cada tipo de átomo tiene una serie específica y finita de energías de excitación, o sea, que tiene una órbita cuántica posible como límite exterior. Cuando la energía absorbida por el átomo alcanza este límite - llamado energía de ionización- el electrón atómico afectado se desprende del átomo y, como consecuencia, el átomo se convierte en un ión positivo. Entonces, como resultado del choque, se tiene un átomo ionizado -al cual le falta un electrón-, además del electrón libre que chocó y del electrón que se desprendió del átomo. Aparte de esta clase de choques, denominados del primer tipo, existe la posibilidad de que se produzcan choques que representan

11 Esto demuestra que la energía requerida para provocar el salto cuántico con seguridad no es el cuanto de energía elemental, ya que existen cantidades menores que dicha energía de resonancia. 
el proceso inverso y, por ello, se llaman del segundo tipo. En este caso, un átomo ya excitado choca con un electrón libre sin transferirle su energía de excitación en forma de radiación, sino que se la traspasa en la forma de energía cinética. Cuando esto sucede, el electrón libre retrocede con una velocidad mayor de la que tenía antes de producirse el choque. Y, por cierto, estos choques del segundo tipo tienen efectos macroscópicos de gran importancia, ya que cuando una radiación pasa a través de un gas, la energía radiante absorbida por las moléculas del gas las excita y se convierte finalmente en energía cinética molecular —es decir, en calor- por medio de los choques del segundo tipo que entonces se producen.

\section{Energía de la cohesión molecular}

Las moléculas de las diversas sustancias químicas - tanto las elementales como las compuestas - están formadas por átomos de los distintos elementos en un número característico y con una ordenación estructural también peculiar para cada sustancia. Por lo tanto, los átomos que constituyen una molécula se encuentran unidos firmemente entre sí y se mantienen relativamente fijos en la posición que les corresponde dentro de la estructura molecular. Desde el punto de vista mecánico, resulta indispensable que se establezca un equilibrio entre las atracciones y las repulsiones de los átomos componentes, para que se mantenga la estructura de la molécula. De acuerdo con las leyes newtonianas del movimiento y con las leyes del electromagnetismo, las fuerzas que mantienen la cohesión molecular son conocidas con el nombre de fuerzas de valencia. Estas fuerzas dependen de la capa exterior de electrones que tiene cada átomo, la cual la integran entre uno y ocho electrones y casi siempre está cargada negativamente $\longrightarrow$ bien, es neutra en el caso de los gases inertes- $y$, por consiguiente, su acción es repelente. Sin embargo, cuando los átomos se aproximan, se puede producir un intercambio de sus electrones exteriores, lo cual provoca un cambio en el signo de la carga de algunos de ellos $y$, por lo tanto, hace que la repulsión se convierta en atracción. Así, por ejemplo, en el caso simple del cloruro de sodio, lo que ocurre es que un electrón de la capa exterior del átomo de cloro abandona dicha capa y pasa a formar parte de la capa exterior del átomo de sodio. De este modo, los dos átomos se ionizan, transformándose en partículas con cargas eléctricas opuestas. Entonces, se forma la molécula de cloruro de sodio, cuya cohesión viene a ser un efecto de la atracción electrostática que se produce entre el átomo de cloro y el átomo de sodio. Cuando se trata de moléculas más complicadas, su cohesión se puede explicar generalmente de manera análoga, aunque con mayor complejidad.

En cambio, el problema es diferente cuando se trata de explicar la interacción que vincula a los dos átomos que constituyen la molécula de hidrógeno; 
porque entre estos dos átomos neutros no puede haber fuerza atractiva, en el sentido newtoniano. En este caso, es indispensable recurrir a la diferencia en el estado cuántico de los respectivos electrones. Para dar una idea de esta diferencia tenemos que traer a cuento el spin de los electrones. Empleando un lenguaje comparativo, podemos decir que las partículas elementales giran como esferas pequeñísimas, unas en un sentido y las otras en el opuesto. Este movimiento produce una cantidad de movimiento angular, a la cual se le denomina spin y puede tener signos opuestos $(+\mathrm{y}-)$. Pues bien, la cohesión de los dos átomos en la molécula de hidrógeno se debe al intercambio que se establece entre los estados cuánticos de sus correspondientes electrones -recordemos que cada átomo de hidrógeno tiene un solo electrón- $y$, por ello, a la causa de este efecto se le llama energía de intercambio. Cuando el spin de estos electrones tiene el mismo signo, la energía de intercambio corresponde a una repulsión $y$, por ende, no es posible que se forme molécula alguna. Mientras que, cuando los spin respectivos son de signo contrario, entonces la energía de intercambio corresponde a una atracción entre los átomos $\mathrm{y}$, en consecuencía, se forma la molécula de hidrógeno. Por lo tanto, la energía de intercambio solamente surge cuando no es nula la probabilidad de que dos partículas de idéntico tipo - como lo son los electrones negativosse encuentren en el mismo lugar del espacio en el mismo instante. Esto es, que únicamente se manifiesta la energía de intercambio en el caso de que dos partículas de la misma especie tengan dominios de presencia posible que encajen el uno en el otro, o sea, cuando poseen la propiedad de homotopía. ${ }^{12}$ Por otra parte, esta posibilidad de que varias partículas elementales ocupen el mismo lugar al mismo tiempo, se relaciona también con el problema de la carencia de individualidad que muestran dichas partículas $\mathrm{y}$, por consiguiente, con la imposibilidad existente hasta ahora de poder identificarlas singularmente de un modo completo. Por lo demás, la energía de intercambio desempeña igualmente una función importante en las acciones recíprocas existentes entre las partículas que integran el núcleo atómico.

\section{Energía interna de los núcleos atómicos}

Los núcleos atómicos son partículas compuestas, integradas por protones positivos y neutrones, salvo en el caso del núcleo de hidrógeno ordinario que está formado por un solo protón. La masa del protón equivale a 1836 veces la masa del electrón, y la masa del neutrón es prácticamente igual; ${ }^{13}$ por lo cual, la masa del átomo se encuentra concentrada, prácticamente, en el núcleo. Por lo tanto, el núcleo atómico está constituido por dos tipos de partículas

12 Véanse nuestros ensayos antes citados; Diánoia, III, 1957, sec. 11, págs. 120-122; Diánoia, IV, 1958, sec. 8, págs. 81-82.

13 En rigor, es de 1837.5 veces la masa del electrón. 
pesadas o bariones, a las que se les ha dado el nombre genérico de nucleones. El protón nuclear tiene una carga positiva cuya magnitud es exactamente igual a la del electrón atómico, pero de signo contrario; mientras que el neutrón es eléctricamente neutro. Por esto, la carga del núcleo siempre es positiva y su magnitud es igual al número de protones que lo forman, e igual también al número de electrones planetarios que tiene el átomo en estado estable. Ahora bien, los neutrones y protones que constituyen un núcleo atómico se encuentran unidos en la forma más poderosa que se conoce $y$, para muchísimos efectos, se comportan como una sola partícula compacta. Esta constitución nuclear planteó el problema de saber cómo pueden unirse así, por un lado, partículas cuya carga eléctrica es del mismo signo y, por otra parte, partículas eléctricamente neutras. La explicación encontrada radica en el llamado defecto de masa de los núcleos atómicos. El protón tiene un peso atómico de 1.00813 unidades y el neutrón tiene un peso atómico de 1.00895 unidades. Naturalmente, el núcleo de hidrógeno ordinario - formado por un solo protón- tiene exactamente el mismo peso atómico del protón y, por ende, es el único núcleo en el que no existe defecto de masa. Pero, en cambio, el núcleo de deuterio o hidrógeno pesado, formado por un protón $\mathrm{y}$ un neutrón, tiene un peso atómico de 2.01472 unidades. Este peso es inferior en 0.00236 unidades a la suma aritmética de las masas del protón y del neutrón, que es de 2.01708 unidades; y, por lo tanto, esa diferencia es su defecto de masa. Por su parte, el núcleo de litio, que consta de tres neutrones y tres protones, tiene un peso atómico de 6.01692 unidades; mientras que la suma de los pesos de sus integrantes es: $3 \times 1.00895+3 \times 1.00813=6.05124$. Por consiguiente, su defecto de masa asciende a 0.03432 unidades. Este incremento en el defecto de masa se va haciendo mayor a medida que se trata del núcleo de un átomo más pesado, hasta llegar a ser de 0.238 unidades para el núcleo de uranio.

Pues bien, como se ha comprobado experimentalmente en los procesos de fusión atómica, lo que ocurre es que al formarse el núcleo de un átomo, una parte de la masa de los nucleones se convierte en energía. La magnitud de la masa transformada corresponde precisamente al defecto de masa y la energía resultante de esta conversión es liberada. En consecuencia, la cohesíón de los protones y los neutrones en el núcleo se debe a la energía faltante que ha sido despedida fuera del propio núcleo. $\mathrm{Y}$, por lo tanto, como se ha verificado igualmente en los experimentos atómicos, para conseguir que un núcleo se desintegre -ya sea por radiactividad natural o provocada artificialmente- es indispensable suministrarle la cantidad de energía que se puso en libertad al formarse el mismo núcleo, para que se opere su conversión inversa en masa y los nucleones separados recuperen la masa que habian cedido. Por supuesto, estas transformaciones recíprocas de masa y energía se realizan de acuerdo con la ecuación de Einstein de la que ya hemos 
hablado antes. Entonces, en el caso de la cohesión nuclear nos encontramos ante el hecho sorprendente de que su causa consiste justamente en la carencia de una cierta cantidad de masa, convertida en energía y puesta en libertad en esta forma. Por lo tanto, no es desacertado el apuntar aquí la posibilidad de que la energía de cohesión nuclear represente una forma de energía verdaderamente opuesta a las otras formas conocidas de la energía; y a la cual se le podría considerar legítimamente como de signo negativo. Sólo que, al mismo tiempo, resulta que esta forma negativa de la energía también es susceptible de convertirse en masa y en cualquier otra de las formas de energía existentes, y viceversa. En todo caso, la energía de cohesión nuclear es la forma más poderosa de energía que se conoce. Para tener una idea, bástanos mencionar que, por su orden de magnitud, equivale a 100 veces la energía electromagnética, a 10000000000000 veces la energía de las llamadas interacciones débiles de las partículas elementales, y a

100000000000000000000000000000000000000

veces la energía gravitacional.

\section{Significación de la energía}

Resumiendo el examen que hemos hecho, tenemos que la energía y la masa son los aspectos primordiales del movimiento, o sea, las formas fundamentales de la existencia objetiva en movimiento. Pero masa y energía no son aspectos que tengan existencia autónoma, sino que, por el contrario, son inseparables entre sí y, a la vez, son inherentes a la existencia material cuya manifestación constituyen. La masa es el aspecto pasivo y la energía es el aspecto activo de la materia, que se mueve incesantemente y se transforma de continuo como resultado de la oposición que se ejerce entre la energía y la masa, puestas de manifiesto en la forma de acción y reacción, de atracción y repulsión, de impulso y resistencia, de cohesión y de desintegración. Además, la energía posee muchas formas que producen efectos diferentes - desplazamiento, rotación, calor, electromagnetismo estático y dinámico, luz, radiaciones invisibles, desintegración de partículas elementales, reacciones químicas, fusión y desintegración nucleares - y cada una de estas formas de energía se transforma en todas las otras en cantidades definidas. Por otro lado, la energía se convierte igualmente en masa, lo mismo que la masa en energía. Así, la suma de todas las masas y de todas las energías es constante; $y$, por lo tanto, también es constante la suma de acciones y reacciones, de atracciones y repulsiones, de impulsos y resistencias, de cohesiones y desintegraciones.

Por otra parte, la categoría de energía forma parte integrante de todos los dominios de la física y, en cierto sentido, lo que constituye el lazo de unión entre todos esos dominios es el principio de la conservación y la trans- 
formación de la energía. En efecto, este principio, expresado como primera ley de la termodinámica, es el punto de partida de la teoría del calor y, a la vez, representa su inseparable unión con la mecánica. De otro lado, los procesos eléctricos y magnéticos o electrodinámicos se encuentran enlazados por medio de varios puentes con los procesos mecánicos, siendo el primero y más importante de estos puentes el principio de conservación de la energía. A su vez, tanto la física relativista como la mecánica cuántica están unidas entre sí y se conectan firmemente con la electrodinámica y la mecánica clásicas, justamente a través del cumplimiento universal que en todos estos campos tiene el principio de la conservación y la transformación de la energía. A más de esto, en todas las aplicaciones técnicas que el hombre hace de sus conocimientos físicos, figura siempre en primer lugar la energía, a tal punto que todo mecanismo $-y$, en general, todo proceso técnico- es juzgado de acuerdo con su balance de energía, o sea, por la proporción en que la energía suministrada es transformada en la forma de energía deseada. Por ello, sin hacer exageración alguna, la energía es considerada como la concepción primordial de la física. Y, con esto, expresamos simplemente la correspondiente importancia, también fundamental, que la energía tiene en la existencia objetiva de la naturaleza y de la sociedad.

Eli de Gontari 